\title{
An atypical manifestation of inguinal endometriosis in the extra pelvic part of the round ligament: a case report
}

\author{
Vito Chiantera $^{1,2}$, Elene Abesadze ${ }^{2}$, Mohamed Gamal Ibrahim ${ }^{3,4}$, Anna Maria Düickelmann ${ }^{4}$, \\ Sylvia Mechsner ${ }^{2} *$
}

\author{
${ }^{1}$ Department of Gynecology, (UNIPA), University of Palermo, Palermo, Italy \\ ${ }^{2}$ Charité University Hospital, Endometriosis Research Centre, Department of Gynaecology, Campus Benjamin \\ Franklin, Berlin, Germany \\ ${ }^{3}$ The University of Münster, Clinic for obstetrics and gynecology, UKM Kinderwunschzentrum, Münster, Germany \\ ${ }^{4}$ Charité University Hospital, Department of Gynaecology, Campus Benjamin Franklin, Berlin, Germany
}

Received: 28 June 2016

Accepted: 05 August 2016

\section{*Correspondence:}

Dr. Sylvia Mechsner,

E-mail: sylvia.mechsner@charite.de

Copyright: (c) the author(s), publisher and licensee Medip Academy. This is an open-access article distributed under the terms of the Creative Commons Attribution Non-Commercial License, which permits unrestricted non-commercial use, distribution, and reproduction in any medium, provided the original work is properly cited.

\begin{abstract}
Establishing the diagnostic and surgical management of the inguinal Endometriosis, with further investigation of the biological character. The imaging findings of CT and PET-CT, biopsy, ultrasound, open surgery of the inguinal region with intraoperative cryosection, confirmation and evaluation of tissue infiltration by endometriosis, laparoscopic removal of all endometriotic lesions, reconstruction of the groin. Based on the history of the 29-year-old patient, suffering from a painful growing induration of the inguinal region. Immunohistochemistry performed, in order to analyze the character of the inguinal endometriosis. CT, PET-CT and biopsy did not confirm the diagnosis of endometriosis. Considering, the progressive symptoms of the patient, was performed the surgical intervention. Open surgery of the inguinal region, with a preparation and separation of the groin fibrotic mass lead to the finding of an affected extra-peritoneal portion of the round ligament. Intraoperative cryosection confirmed endometriosis. Simultaneous laparoscopy showed peritoneal endometriosis (rASRM I) and an alteration of the inner round ligament, involving the inner inguinal channel in this process. All endometriotic lesions were removed and the inguinal region reconstructed. The immunohistochemical staining gave evidence of the endometriotic tissue, surrounded by smooth muscle metaplasia. We consider that, reporting this rare case of endometriosis, based on a case report and a literature review, affecting intra and extra peritoneal portion of the round ligament, is an important aid to avoid a wrong diagnosis and method of therapy in future. Our data demonstrated the fully recovery of the patient, after surgical treatment, reporting symptom-free status.
\end{abstract}

Keywords: Extra genital endometriosis, Endometriosis, Affecting the intra and extra-peritoneal portion of the round ligament, Inguinal endometriosis, Smooth muscle metaplasia

\section{INTRODUCTION}

Endometriosis is a common disease affecting millions of women during the reproductive age. ${ }^{1,2}$ The typical localisation of endometriosis is the intra-abdominal cavity, with manifestation of endometriotic lesions in the pelvis affecting genital organs (so-called endometriosis genitalis externa and interna). Nevertheless, there are extra-genital manifestations in $15 \%$ of the cases; for example, in the bowel or the diaphragm. ${ }^{26,34}$ Most of those lesions appear intra-abdominally and only $0.5-1 \%$ extra-abdominally. There are descriptions of endometriosis in scars after surgery, with secondary dissemination of endometriotic tissue or primary and secondary development of endometriosis in the 
umbilicus, in the lung, brain, or the inguinal region. 1,13,16,18,21,29 Remarkably, the establishment of endometriosis in the extra-peritoneal part of the round ligament was found to be $0.3-0.6 \%$ among the extraabdominal manifestation. ${ }^{1}$ In general, it seems to affect only $0.07 \%$ of the patients with endometriosis. ${ }^{30}$ Inguinal endometriosis is established rarely and so far, there appears to be no evident based strategy available for diagnostic or the therapeutic management.

This study will present the evidence of inguinal mass, representing the methods of diagnosis and therapeutic treatment.

\section{CASE HISTORY}

A 29 year-old nulliparous woman was presented to our clinic, suffering from the swelling of a right inguinal area over a two-year period. The complaints used to increase cyclically, simultaneously to the menstrual bleeding. The induration was very painful, especially during the menstruation. Patient had regular menstrual cycle, without endometriosis-related symptoms, like dysmenorrhoea, pelvic pain, dyschezie or dyspareunia. No surgical treatment was performed previously, as well.

\section{Diagnostic findings}

During medical examination, palpation showed a $2 \times 2$ $\mathrm{cm}$ diameter tumour disclosed in the right inguinal area, laterally on the symphysis pubis. As concerning underlying structures, they were relatively fixed and immobile. The palpation was very painful, no evidence of swelling in the contralateral side. Significant was, that the gynaecological examination revealed no pathology in pelvic area. Pelvic ultrasound was unremarkable, but an inguinal ultrasound revealed a low echoic mass of more than $2 \mathrm{~cm}$ diameter (Figure 1).

Within the medical history of the patient we could see, that orthopaedics, in order to clarify and diagnose the uncommon condition, performed a fine needle biopsy of the inguinal mass. Histology revealed fibrosis. Further imaging with CT scan and PET-CT gave no affect in clarification of the uncommon mass.

\section{Surgery}

According to these complaints and findings, the patient was considered to undergo an excision of the inguinal mass, along with a laparoscopy. First step was an inguinal excision, which revealed the $2 \times 2 \mathrm{~cm}$ diameter mass, attached to the extra-peritoneal part of the round ligament, superolaterally to the right pubic tubercle. The excised specimen sent to pathology indicated endometriosis. The further preparation processed in the direction of the deep inguinal ring and new frozen sections justified endometriosis repeatedly. Subsequently, the inguinal region was closed temporarily.
Second part of the surgery was performed laparoscopically. Endometriosis was compatible to the stage rASRM I. The intra-abdominal part of the round ligament was infiltrated with endometriotic lesions, therefore completely retracted into the right inguinal channel; likewise, the uterus was shifted to the right pelvic wall. On the left ovarian fossa and Sacrouterin ligament were represented typical black cicatricle endometriotic lesions, which were excised subsequently. Considering wide infiltration approximately was resected one-third of the abdominal part of the round ligament.

For further manipulation, laparoscopy and open surgery have been combined, namely performed simultaneously. The residual part of the round ligament, proximal to the uterus, was embed medially to the superficial inguinal ring and through the open groin connected to the superior wall of the inguinal channel, followed by the fixation at the transversal fascia.

As a final step, an inguinal channel was reconstructed for a hernia prophylaxis, with a Vicryl mesh, followed by consecutive closure of the epidermis the last laparoscopic imaging showed mobile uterus, slightly shifted to the dextra position. Essentially related to the normal anatomic topography (Figure 2A-I).

\section{Immunohistochemistry}

Tissue preparation: All excised lesions were directly fixed in $4 \%$ buffered formalin for $12 \mathrm{~h}$ and then embedded in paraffin. Serial sections (1-2 $\mu \mathrm{m}$ thick) were used for haematoxylin-eosin staining and immunohistochemistry.

After deparaffinization in xylene $(2 \times 5 \mathrm{~min}$ at room temperature) and rehydration (10 $\mathrm{min}$ in acetone and acetone/Tris-buffered saline (TBS)) at a 1:1 ratio, likewise only in TBS (at room temperature), the heatinduced epitope retrieval (HIER) procedure was performed by heating the specimens in citrate buffer $(0.1$ $\mathrm{M}$ citric acid and $0.1 \mathrm{M}$ sodium citrate, $\mathrm{pH} 6.0$ at $700 \mathrm{~W}$ in a microwave oven for $17 \mathrm{~min}$ ). Afterwards sections were blocked with $10 \%$ foetal calf serum (FCS) for 30 min at room temperature.

Oestrogen receptor (ER) and progesterone receptor (PR) were performed using the primary antibodies against ER (clone 1D5, dilution 1:40) and PR (clone 1A6, 1:100) obtained from DakoCytomation, Glostrup, Denmark. The alkaline-phosphatase-anti-alkaline phosphatase complex (APAAP) method was used for detection and analysed by Fast Red as the chromogen.

Smooth muscle Actin (smActin) and desmin analysis: The sections were incubated for $1 \mathrm{~h}$ at room temperature with the monoclonal mouse anti-smActin or monoclonal mouse anti-desmin antibody (dilution 1:50, Dako, Hamburg, Germany), followed by incubation with a Cy2conjugated donkey anti-mouse antibody (dilution 1:100, Dianova, Hamburg, Germany) for $45 \mathrm{~min}$. 
There was used the uterine tissue as a positive control. All negative control sections were processed by omitting the primary antibody. Staining was detected using a radiophoto microscope (Carl Zeiss, Göttingen, Germany). Pictures were taken in different magnifications (40, 100 or 1000x) and further processed using the Adobe Photoshop (Adobe Systems, Unterschleissheim, Germany).

In order to investigate the biological character of inguinal endometriosis, there was performed immune histochemical analysis against ER and PR, likewise smooth muscle differential markers: smooth muscle actin and desmin.

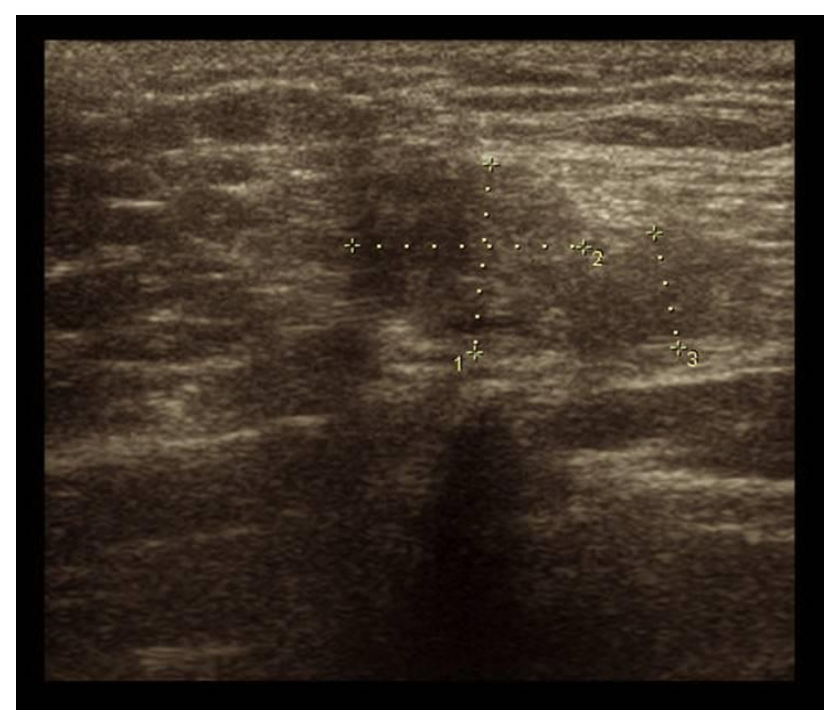

Figure 1: Trans abdominal ultrasonography of right inguinal region. Two solid hypoechoic round lesion of $2 \times 1,4 \mathrm{~cm}$ and $0,9 \times 0,7 \mathrm{~cm}$, with irregular morphology and blurred margins, which are located in the lower right anterior abdominal wall.

The immune histochemical staining revealed typical endometriotic lesions (epithelial and stromal cells) (Figure 3). A strong expression of oestrogen and progesterone receptors were detected in lesions (Figure $3 \mathrm{~A}$ and $\mathrm{B}$ ). Furthermore, the identification of the smooth muscle actin, likewise desmin-positive cells in the endometriotic associated surrounding tissue proves evidence of smooth muscle metaplasia (Figure 3C and D). Nonetheless, there was a divergence, between the expression of smooth muscle actin (marker for undifferentiated smooth muscle cells) and desmin (a marker molecule for differentiated smooth muscle cells), which is only expressed in mature smooth muscle cells.

\section{Postoperative follow-up and management}

After two-month postoperative period, the patient was presented in our clinic, completely free from complaints: no further cyclical painful irritations in the inguinal region. Considering, patient's actual seeking fertility, shortly after discharge, no adjuvant hormonal treatment was recommended.

\section{DISCUSSION}

Until today, approximately 100 cases of the round ligament endometriosis are reported and further 150 case reports, concerning inguinal endometriosis. Cullen described the first case of 'Adenomyom' of the round ligament in 1896. Throughout the following years, eventually 20 similar cases were described, analysed and interpreted by Robert Meyer. ${ }^{20}$ Although this phenomenon had already been described in many instances, inguinal endometriosis still stays a rare form of extra-genital endometriosis, consequently standardized treatment options are missing.

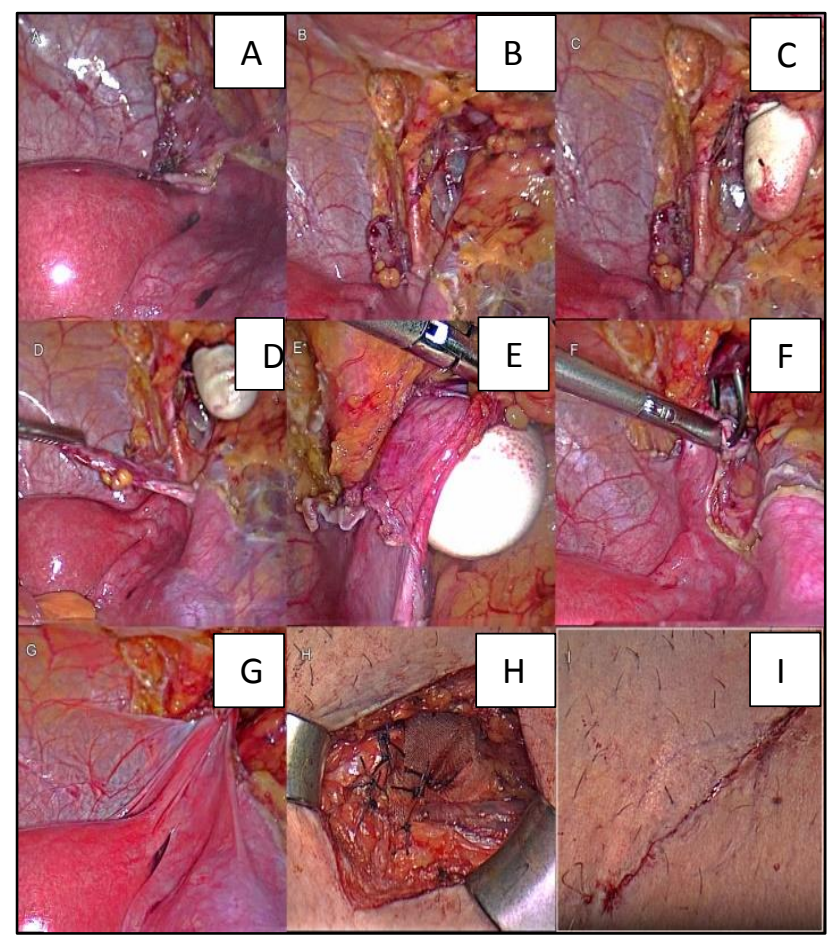

Figure 2 (A) (B): Laparoscopic part of the operation: Status after inguinal excision, processed in the direction of the deep inguinal ring, removal of $2 \times 2$ cm diameter mass, attached to the extra-peritoneal part of the round ligament. Resection of the round ligament, approximately one-third of the abdominal part, considering the wide infiltration. $(C)(D)$ :

Combination of the laparoscopy and open surgery.

$(E)(F)$ : Fastening the residual part of the round

ligament, proximal to the uterus, medially to the superficial inguinal ring and going through the open groin, connected to the superior wall of the inguinal channel, followed by the fixation at the transversal fascia. (G) The last laparoscopic imaging showing mobile uterus, slightly shifted to the dextra position, related to the normal anatomic topography. $(\mathrm{H})$ Reconstruction of the inguinal channel as a hernia prophylaxis, with a Vicryl mesh. (I) Followed by consecutive closure of the epidermis. 


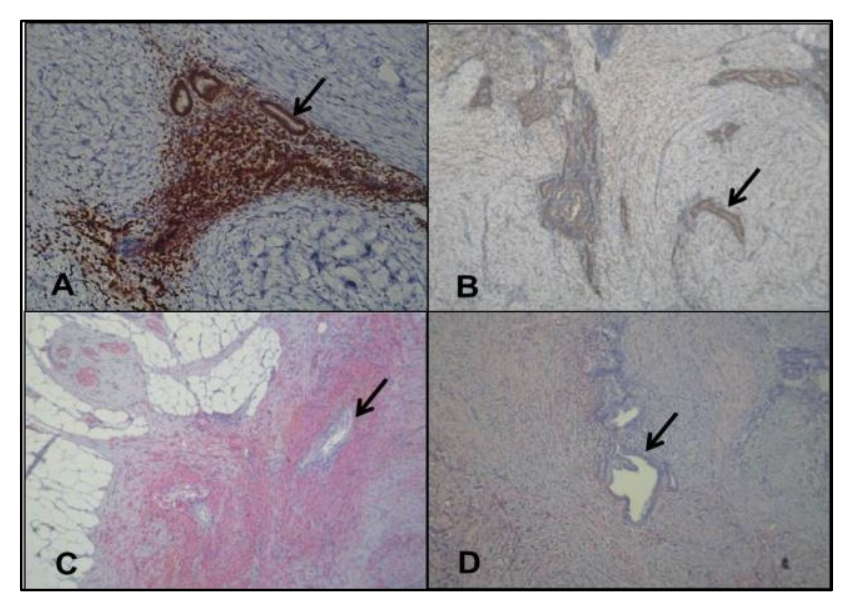

Figure 3: Immunohistochemical characterization of the right inguinal endometriosis. When endometriotic glands (arrow) and stroma express (A) oestrogen receptor, only glandular epithelium expresses (B) progesterone receptors. Smooth muscle metaplasia is evident as endometriotic stromal cells express (C) alpha smooth muscle actin. Furthermore, some stroma cells are seen expressing (D) desmin as a marker for differentiated smooth muscle cells; X200.

Interestingly, a retrospective analysis of women undergoing laparoscopy for treatment of deep infiltrating endometriosis (DIE) demonstrated a prevalence of endometriosis of the round ligament of $13.8 \%$. $^{7}$ They still removed the inner part of the round ligament, even for cases of macroscopic alterations; such as shortening, deviation or thickening for a histopathological analysis. In the light of this, such an endometriosis manifestation seems to be underestimated, and a rigorous evaluation of the structure must now become part of the routine surgical treatment of patients with endometriosis. ${ }^{7}$

The typical history of cyclical symptoms is the main indication of endometriosis and leads to the correct diagnosis. Patients were suffering from cyclical inguinal swelling and catamenial pain. Sometimes the pain changed into the permanent pain. CT and PET-CT imaging, like wise fine needle biopsy resulted no significant impact on diagnostic evaluation. Based on other works, inguinal ultrasound appeared to be the most profitable way to detect an echogen nodule, therefore provides better opportunity to monitor the lesion. ${ }^{4,11}$ Furthermore, this method allows a differentiation from other inguinal pathological findings, alike inguinal hernia or enlarged lymph nodes. Additionally, in some selected cases, magnet resonance imaging has been presented to be particularly effective method in diagnosis of the extraperitoneal endometriosis. ${ }^{4,14,17,33}$ However, MRI is not obligatory when the lesion is previously detectable by the ultrasound. As a final consensus, it requires a surgical excision and histological examination to give the definitive diagnosis. The surgical treatment should involve an adequate excision of the intra and extraperitoneal part of the round ligament to avoid disease recurrence. ${ }^{10}$
It is remarkable, that in most cases of the inguinal endometriosis extra-peritoneal part of the round ligament is affected. ${ }^{6}$ Rarely, endometriosis also have been observed in inguinal hernial sacs in lymph nodes, in canal of Nuck or in regions associated with femoral vessels and femoral hernia. ${ }^{2,3,24,27,28,32}$

Subsequent to extra-peritoneal manifestations of endometriosis, a simultaneous laparoscopy is strongly recommended, in order to exclude further expression of intraperitoneal endometriosis. This case, based on the example of our patient, is clear evidence of this theory. Postoperative results were remarkably excellent. Patient was free from previous complaints and from further endometriosis-associated symptoms. The quality of life was apparently improved. Generally, inguinal endometriosis usually negate further endometriosisassociated symptoms like dysmenorrhoea or pelvic pain. In order to improve and investigate new methods of treatment and be able to remove endometriotic lesions totally, we had to find the right plain of endometriosisfree tissue within the round ligament. Therefore, intraoperatively frozen sections of endometriotic lesions were sent to the pathology to explore the infiltration, in order to come up with the right target. This concept improves the possibility to excise the affected tissue completely and avoid further recurrence.

The pathway, mentioned above, is the direct extension along the round ligament from the pre-existing endometriosis in the pelvis. ${ }^{6}$ In our case, infiltration of the intraabdominal part was accompanying the round ligament into the inguinal canal, involving the extraperitoneal part. In $32 \%$ of the described cases, infiltration affected an inguinal hernia, occasionally, even after excluding the intraperitoneal endometriosis laparoscopically. ${ }^{6,25}$ Nonetheless, in previous six cases the initial tentative diagnosis of inguinal hernia were reported, the confirmation of this diagnosis throughout the surgery was missing. ${ }^{5}$ In all of these women, the endometriotic lesions were located in the extra-peritoneal portion of the round ligament, without any apparent spatial communication with the pelvis. Consequently, the statement of Meyer in 1930, about the embryological development of the round ligament, can be considered very interesting. It declares that the second pathway is a reason of endometriosis dissemination, according to the lymphatic spread. Recently Moore, J. G have suggested the theory considering that retroperitoneal endometriotic lesions are probably associated with a lymphatic spread of endometrial cells. ${ }^{23}$ This could be lymphatic vessels, which have originated in the uterus and run along the round ligament. ${ }^{15}$ Accordingly, this could be a possible explanation of endometriosis in the extra-peritoneal part of the round ligament, whereas the intraperitoneal endometriosisis is not emerged. In case of intraperitoneal endometriosis, a crossing of the endometriotic cells through the peritoneal mesothelium into the lymphatic and venous system of the round ligament seems to be possible. ${ }^{10}$ Although it has been confirmed that a 
lymphatic spread in retroperitoneal deep infiltrating endometriosis is in fact a frequent phenomenon, the inguinal endometriosis is not typically localised in the inguinal lymph nodes but rather in the round ligament. ${ }^{19}$

In the literature, it has also been reported that in $90 \%$ of the cases preferred manifestation of inguinal endometriosis involves the right groin. ${ }^{6}$ Why this affection involves predominantly the right side of the groin is still undefinable. Furthermore, usually we inspect more right sided affection of the diaphragmatic endometriosis. The intraperitoneal circulation of the fluid could explain this, assuming that endometriotic cells (from transtubal flow or pelvic endometriosis) remain in the region of the right round ligament for a longer period, than in the left side. In addition, the sigmoid seems to protect the left round ligament. Therefore, the persistence of such cells in the right deep inguinal ring and the inferior epigastric vessels could favour the passage of these cells across the mesothelium into the lymph system of the right round ligament resulting in a consecutive development of endometriosis. ${ }^{5}$

Based on the long-term follow-up, there were observed late complications - the malignant transformation under oestrogen replacement therapy, especially in case of hysterectomy, while Patients were taking oestrogen replacement monotherapy for the menopausal syndrome. ${ }^{9,22}$ Regarding such complications, it is very important to remove the inguinal mass totally.

For further analysis, were performed the biological properties of this inguinal endometriosis, immunohistochemical examinations. Absolutely all epithelial and stromal cells express an oestrogen and progesterone receptor, proving a high hormonal sensitivity of the endometriotic lesion. Besides the hormonal sensitivity, there is also a high COX-2 expression, which reflects the high activity of inguinal lesions. $^{31}$ Another famous factor, associated with the activity of endometriotic lesions is the metaplasia process. ${ }^{18}$ In this context, we analyzed character of the rarely found inguinal endometriotic lesion.

However, in the endometriotic lesion was a high expression of smooth muscle actin, a general marker molecule for smooth muscle cells and myofibroblasts, indicating a high content of smooth muscle cells. ${ }^{18}$ In serial sections, was expressed desmin (a marker molecule for differentiated smooth muscle cells) in corresponding with smooth muscle cells (Figure 3). However, on another hand, discrepancy was detected, between the expression of smooth muscle actin and desmin, expressed only in mature smooth muscle cells. This discovery indicates, that the smooth muscle cells were of new origin in the differentiation process. ${ }^{8,18}$

In summary, transvaginal and inguinal ultrasound was the method of choice to investigate the extension of an inguinal endometriosis. The laparoscopy should be performed, in order to exclude or treat a pelvic endometriosis. Recurrence is definitely rare after a complete and adequate surgical treatment (including the intra and extra-peritoneal part of the round ligament). However, in context with the biological properties of the inguinal endometriotic lesion, a postoperative endocrine treatment can be discussed for recurrence prevention or in a case of recurrent disease. ${ }^{5}$

\section{CONCLUSION}

We consider that, reporting this rare case of endometriosis, based on a case report and a literature review, affecting intra and extra peritoneal portion of the round ligament, is an important aid to avoid a wrong diagnosis and method of therapy in future. Our data demonstrated the fully recovery of the patient, after surgical treatment, reporting symptom-free status.

\section{Funding: No funding sources}

Conflict of interest: None declared

Ethical approval: The study was approved by the IRB of Charité ethics committee (EA)

\section{REFERENCES}

1. Ali Kemal Uzunlar FY, Mehmet Y, Özden K, Mehmet Ö. Inguinal Endometriosis: a Report of Two Cases and a Review of the Literature. Turk J Med Sci. 2000:389-92.

2. Brzezinski A, Durst AL. Endometriosis presenting as an inguinal hernia. Am J Obstet Gynecol. 1983;146:982-3.

3. Bungart. Inguinal endometriosis. Arch $f$ klin. Chir.1925.

4. Calo PG, Esu F, Tatti A. Isolated inguinal endometriosis. Case report with ultrasonographic preoperative diagnosis. G Chir. 2011;32:263-5.

5. Candiani GB, Vercellini P, Fedele L. Inguinal endometriosis: pathogenetic and clinical implications. Obstetrics and gynecology. 1991;78:191-4.

6. Clausen I, Nielsen KT. Endometriosis in the groin. Int J Gynaecol Obstet. 1987;25:469-71.

7. Crispi CP, De Souza CA, Oliveira MA. Endometriosis of the round ligament of the uterus. $\mathrm{J}$ Minim Invasive Gynecol. 2012;19:46-51.

8. Ebert AD, Rosenow G, David M. Co-occurrence of atypical endometriosis, subserous uterine leiomyomata, sactosalpinx, serous cystadenoma and bilateral hemorrhagic corpora lutea in a perimenopausal adipose patient taking tamoxifen (20 $\mathrm{mg}$ /day) for invasive lobular breast cancer. Gynecol Obstet Invest. 2008;66:209-13.

9. Elemenoglou J, Skopelitou A, Nomikos I. Carcinoma of the inguinal region arising from endometriosis of the round ligament. Report of a case. Eur J Gynaecol Oncol. 1993;14:28-32. 
10. Fedele L, Bianchi S, Frontino G. Radical excision of inguinal endometriosis. Obstetrics and gynecology. 2007;110:530-3.

11. Freed KS, Granke DS, Tyre LL. Endometriosis of the extraperitoneal portion of the round ligament: US and CT findings. J Clin Ultrasound. 1996;24:540-2.

12. Giudice LC.Clinical practice. Endometriosis. N Engl J Med. 2010;362:2389-98.

13. Hong YJ, Paik HC, Kim HJ. A case of parenchymal pulmonary endometriosis. Yonsei Med J. 1990;40:514-7.

14. Kirkpatrick A, Reed CM, Bui-Mansfield LT. Radiologic-pathologic conference of Brooke Army Medical Center: endometriosis of the canal of Nuck. AJR Am J Roentgenol. 2006;186:56-7.

15. Kratz. Anatomy of the female reproductive system. Current obstetric and gynecologic diagnosis and treatment. $6^{\text {th }}$ ed. Norwalk, Connecticut: Apple-ton and Lange; 1987:1-46.

16. Latcher JW. Endometriosis of the umbilicus. Am J Obstet Gynecol. 1953;66:161-8.

17. Licheri S, Pisano G, Erdas E. Endometriosis of the round ligament: description of a clinical case and review of the literature. Hernia. 2005;9:294-7.

18. Mechsner S, Bartley J, Infanger M. Clinical management and immunohistochemical analysis of umbilical endometriosis. Archives of gynecology and obstetrics. 2009;280:235-42.

19. Mechsner S, Weichbrodt M, Riedlinger WF. Estrogen and progestogen receptor positive endometriotic lesions and disseminated cells in pelvic sentinel lymph nodes of patients with deep infiltrating rectovaginal endometriosis: a pilot study. Human reproduction. 2008;23:2202-9.

20. Meyer RM. AdenoWbrosis und Adenomyosis am Nabel. Handbuch der Gynäkologie. von Stöckel, Berlin. 1930;3:511-5.

21. Michowitz M, Baratz M, Stavorovsky M. Endometriosis of the umbilicus. Dermatologica 1983;167:326-30.
22. Milam MR, Atkinson JB, Currie JL. Adenosarcoma arising in inguinal endometriosis. Obstetrics and gynecology. 2006;108:753-5.

23. Moore JG, Binstock MA, Growdon WA. The clinical implications of retroperitoneal endometriosis. Am J Obstet Gynecol. 1988;158:1291-8.

24. Moore WR. Inguinal endometriosis in bilateral hernia sacs associated with extensive pelvic endometriosis. Harper Hosp Bull. 1957;15:242-5.

25. Quagliarello J, Coppa G, Bigelow B. Isolated endometriosis in an inguinal hernia. Am J Obstet Gynecol. 1985;152:688-9.

26. Redwine DB. Attachment of endometrial cells to peritoneum--facilitated? Fertil Steril. 2001;76:421-2.

27. Sampson JEA. Peritoneal Endometriosis due to the menstrual dissemination of endometrial tissue into the peritoneal cavity. American Journal Of Obstetrics And Gynecology. 1927;14:93-4.

28. Scaglione V, Granieri A. [Bilateral endometriosis of the canal of Nuck simulating strangulated hernia. Ann Ostet Ginecol Med Perinat. 1981;102:55-61.

29. Singh KK, Lessells AM, Adam DJ. Presentation of endometriosis to general surgeons: a 10-year experience. Br J Surg. 1995;82:1349-51.

30. Sun ZJ, Zhu L, Lang JH. A rare extrapelvic endometriosis: inguinal endometriosis. J Reprod Med. 2010;55:62-6.

31. Terada S, Miyata Y, Nakazawa H, Higashimori T, Arai T, Kikuchi Y, et al. Immunohistochemical analysis of an ectopic endometriosis in the uterine round ligament. Diagn Pathol. 2006;1:27

32. Theander G, Haeger K. Angiography in the Diagnosis of Inguinal Endometriosis. Acta Radiol Diagn (Stockh). 1964;2:100-4.

33. Tokue H, Tsushima Y, Endo K. Magnetic resonance imaging findings of extrapelvic endometriosis of the round ligament. Jpn J Radiol. 2009;27:45-7.

34. Victory R, Diamond MP, Johns DA. Villar's nodule: a case report and systematic literature review of endometriosis externa of the umbilicus. J Minim Invasive Gynecol. 2007;14:23-32.

Cite this article as: Chiantera V, Abesadze E, Ibrahim MG, Dückelmann AM, Mechsner S. An atypical manifestation of inguinal endometriosis in the extra pelvic part of the round ligament: a case report. Int J Reprod Contracept Obstet Gynecol 2016;5:3202-7. 